\title{
In vitro evaluation of an alternative method to bond molar tubes
}

\author{
Célia Regina Maio PINZAN-VERCELINO¹, Arnaldo PINZAN², Júlio de Araújo GURGEL³ ${ }^{3}$ Fausto Silva BRAMANTE¹, \\ Luciana Maio PINZAN ${ }^{4}$
}

\author{
1- DDS, MSc, PHD, Assistant Professor, Department of Orthodontics, University Center of Maranhão (UNICEUMA), São Luís, MA, Brazil. \\ 2- DDS, MSc, PhD, Associate Professor, Department of Pediatric Dentistry, Orthodontics and Community Health, Bauru School of Dentistry, University of São \\ Paulo, Bauru, SP, Brazil. \\ 3- DDS, MSc, PhD, Professor and Program Director of Master in Orthodontics, University Center of Maranhão (UNICEUMA), São Luís, MA, Brazil. \\ 4- Undergraduate student, Clinical practice, Bauru, SP, Brazil.
}

Corresponding address: Dr. Célia Regina Maio Pinzan-Vercelino - Alameda dos Sabiás, 58 - Portal dos Pássaros - Boituva - SP - $18550-000$ - Brazil - Phone: 551533635958 - Fax: 551432271282 - e-mail: cepinzan@hotmail.com

Received: May 25, 2009 - Modification: November 05, 2009 - Accepted: February 16, 2010

\section{ABSTRACT}

espite the advances in bonding materials, many clinicians today still prefer to place
bands on molar teeth. Molar bonding procedures need improvement to be widely
accepted clinically. Objective: The purpose of this study was to evaluate the shear bond
strength when an additional adhesive layer was applied on the occlusal tooth/tube interface
to provide reinforcement to molar tubes. Material and methods: Sixty third molars were
selected and allocated to the 3 groups: group 1 received a conventional direct bond followed
by the application of an additional layer of adhesive on the occlusal tooth/tube interface,
group 2 received a conventional direct bond, and group 3 received a conventional direct
bond and an additional cure time of $10 \mathrm{~s}$. The specimens were debonded in a universal
testing machine. The results were analyzed statistically by ANOVA and Tukey's test ( $\alpha=0.05$ ).
Results: Group 1 had a significantly higher ( $p<0.05$ ) shear bond strength compared to
groups 2 and 3 . No difference was detected between groups 2 and $3(p>0.05)$. Conclusions:
The present in vitro findings indicate that the application of an additional layer of adhesive
on the tooth/tube interface increased the shear bond strength of the bonded molar tubes.

Key words: Molar. Shear strength. Dental bonding.

\section{INTRODUCTION}

Presently, clinicians are concerned about the clinical effectiveness of the orthodontic procedures. Orthodontists, patients and patients' parents want to obtain the best results in the shortest time. There are many factors that influence treatment duration, including characteristics associated with the problem, such as amount of anteroposterior or vertical discrepancy; age; patient compliance; psychological implications and treatment efficiency degree $5,21,23,26,27$. Besides, rebonding of the attachments and recementation of the bands should have been considered, which are undesirable in a private clinic because they are costly and time-consuming procedures ${ }^{18,20}$.

In contemporary orthodontic practice, direct bond in molars is a less frequently adopted practice, in spite of its advantages: it reduces chairside time to assemble the orthodontic fixed appliance, favors the maintenance of periodontal health, eliminates the spaces caused by the bands, facilitate the detection of caries and provides a better esthetics ${ }^{2,29}$. A study ${ }^{9}$ performed in the United States showed that only 22$30 \%$ of the orthodontists usually bond the molars. This fact is likely related to the clinical failure rate of this procedure.

It has been extensively demonstrated ${ }^{13,15,19,28}$ a clinical failure rate of bonded molars of $14.8 \%$ at least. However, no emphasis is given in the literature to the loose bands, except for one study ${ }^{2}$ that compared bonded and banded molars. Boyd and Baumrind $^{2}$ (1992) evaluated banded and bonded molars in adolescents and adults, and observed that banded maxillary molars in both age groups showed significantly more loss of attachment during treatment than bonded maxillary molars; they also found that bonded molars in the lower arch failed 
more frequently than banded ones.

In spite of advances in orthodontic bond materials, there is still a need to improve current bonding procedures ${ }^{20}$, especially for teeth that are subjected to higher occlusal forces, like the molars ${ }^{22}$.

The aim of this study was to evaluate if the application of an additional adhesive layer on the occlusal teeth/tube interface provides reinforcement to molar tubes.

\section{MATERIAL AND METHODS}

Approval of this protocol was received from the institutional review board prior to the start of the study (Protocol 00951/08).

Sixty human mandibular third molars with indication for surgical removal were selected. The criteria for tooth selection included the following: intact buccal enamel not subjected to any pretreatment chemical agents, e.g., hydrogen, no cracks from the presence of the extraction forceps, no caries, and no abrasion or any other crown defect.

The teeth were obtained from a private practice, and were stored in a refrigerated chloramine-T solution for decontamination. Afterwards, the teeth were placed in PVC rings with self-curing acrylic resin; the long axis of each tooth was vertical, and only the crowns were exposed. Each tooth was mounted with the buccal surface of the crown positioned perpendicularly to the PVC ring base with the help of an acrylic squadron, to facilitate the mechanical tests. After resin polymerization, all specimens were immediately immersed in distilled water.

The specimens were randomly divided into 3 groups of 20 teeth each. Each group underwent a different bond protocol: group 1 (experimental) underwent conventional direct bonding followed by the application of an additional layer of adhesive on the occlusal tooth/tube interface, and $10 \mathrm{~s}$ of lightcure; group 2 (control 1) underwent conventional direct bonding only; and group 3 (control 2) underwent conventional direct bonding followed by an interval of $40 \mathrm{~s}$, and additional $10 \mathrm{~s}$ of light-cure.

Before the direct bond, the buccal surface of each tooth was polished for $10 \mathrm{~s}$ with pumice and rubber prophylactic cups with a low-speed handpiece. The surface was then rinsed under water and dried with an oil-free air spray for $30 \mathrm{~s}$. The prophylactic cups were changed after every 10 teeth to obtain a better procedure pattern. The teeth were then etched with $37 \%$ phosphoric acid, for $30 \mathrm{~s}$, rinsed with an air-water spray for 30 more $s$ and air-dried until a proper white frosted appearance was observed. The next step was the application of a thin coat of primer (Transbond XT Primer; 3M
Unitek Orthodontic Products, Monrovia, CA, USA), followed by the direct bonding of the single tube bracket convertible for bonding (Morelli Ortodontia, Sorocaba, SP, Brazil, base area of $13.6 \mathrm{~mm}^{2}$ ), using the Transbond XT adhesive. The tubes were kept in the manufacturer's packaging until immediately prior to bonding and they were handled at all times with bonding tweezers to avoid any contamination of the bonding base, which could interfere with the results. The adhesive was placed on the tube base, and the tube was pressed firmly against the tooth surface to secure a thin layer of adhesive and to pattern the procedure ${ }^{15}$. After removal of excess adhesive from around the attachment, the adhesive was light-cured at a distance of approximately 5 $\mathrm{mm}$ above the interproximal contact for $20 \mathrm{~s}$ (10 s on each side), according to the manufacturer's instructions.

The described procedure was followed for group 2 (Figures $2 \mathrm{~A}$ and $2 \mathrm{~B}$ ), but the specimens in the other groups were prepared with some technical differences.

In group 1, immediately after the conventional
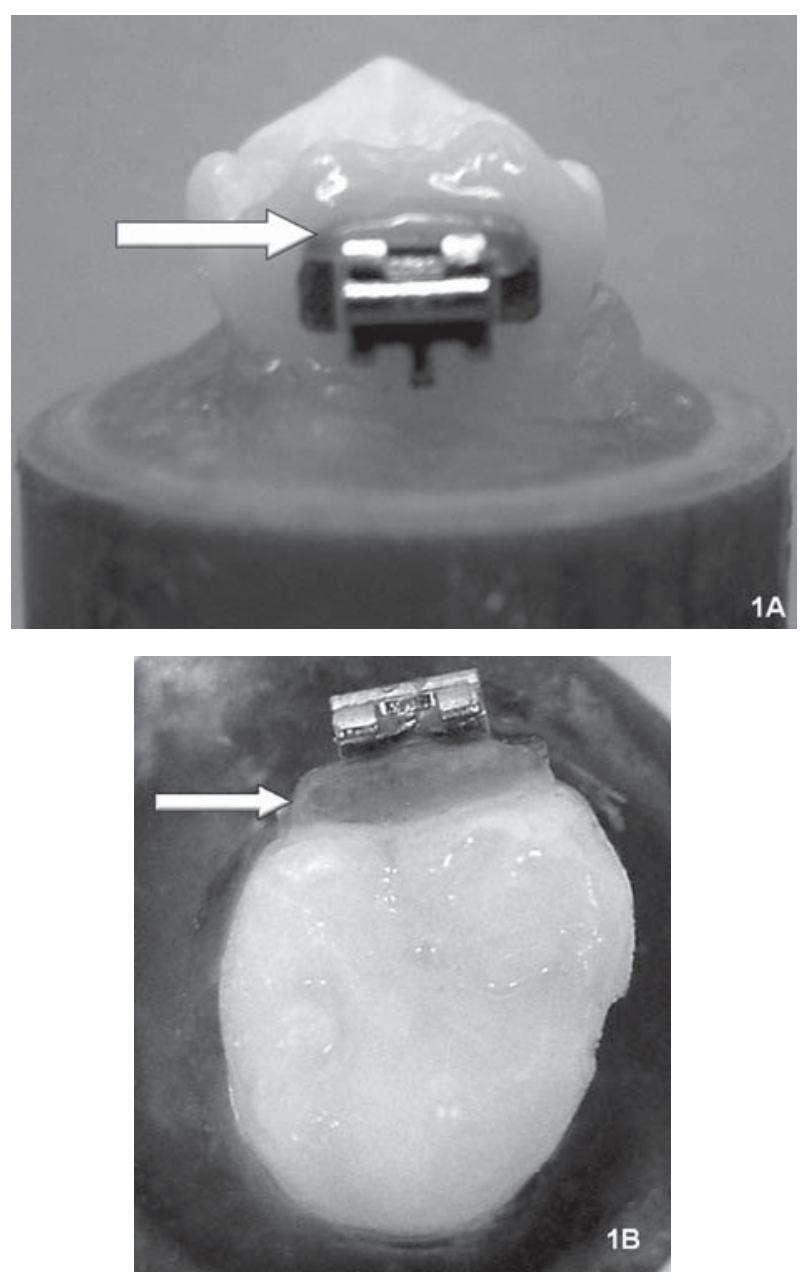

Figure 1-Specimen preparation. Group 1: with application of an additional layer of adhesive in the occlusal teeth/tube interface (A: vestibular view; B: occlusal view) 
direct bond was performed, an additional layer of adhesive was applied on the occlusal tooth/tube interface, followed by 10 additional s of light-cure (Figures $1 \mathrm{~A}$ and $1 \mathrm{~B}$ ). To standardize the quantity of the applied adhesive, a metal spatula with a demarcation line at $2 \mathrm{~mm}$ was used in order to avoid extreme adhesive plantation. The adhesive tube was pressed firmly to this line. The adhesive was applied using microbrush tips, only close to the bond interface, without extending beyond the attachment limits.
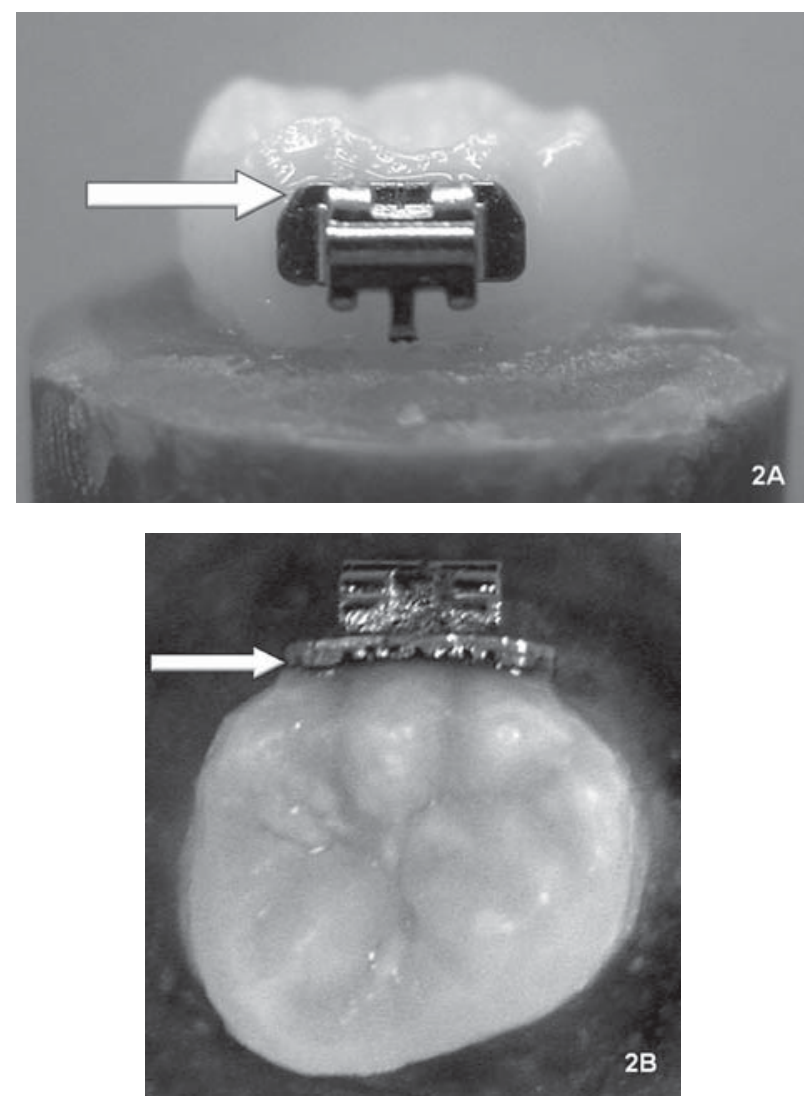

Figure 2- Specimen preparation. Group 2 and Group 3: conventional direct bond (A: vestibular view; B: occlusal view)

Table 1- Bond strength data [means, standart deviation (SD), median, minimum and maximum values], in MPa and in kilograms $(\mathrm{Kg})$

\begin{tabular}{lcccccc}
\hline & \multicolumn{2}{c}{$\begin{array}{c}\text { Group 1 } \\
\text { (experimental) }\end{array}$} & \multicolumn{2}{c}{$\begin{array}{c}\text { Group 2 } \\
\text { (control 1) }\end{array}$} & \multicolumn{2}{c}{$\begin{array}{c}\text { Group 3 } \\
\text { (control 2) }\end{array}$} \\
\hline Mean & $\mathrm{MPa}$ & $\mathrm{Kg}$ & $\mathrm{MPa}$ & $\mathrm{Kg}$ & $\mathrm{MPa}$ & $\mathrm{Kg}$ \\
$\mathrm{SD}$ & $17.08^{\mathrm{A}}$ & 23.69 & $12.53^{\mathrm{B}}$ & 17.37 & $12.60^{\mathrm{C}}$ & 17.48 \\
Median & 3.28 & 4.55 & 2.09 & 2.9 & 1.97 & 2.74 \\
Minimum & 16.35 & 22.66 & 13.25 & 18.38 & 13.1 & 18.16 \\
\hline Maximum & 11.68 & 16.2 & 8.65 & 12.01 & 8.38 & 11.63 \\
\hline
\end{tabular}

Means followed by different uppercase letters differ significantly (ANOVA and Tukey's test; $p<0.05$ )
In group 3, the specimens were light cured for an additional $10 \mathrm{~s}$ from the occlusal side.

All steps were performed by a single person throughout the experiment, and all the intervals were controlled by use of a chronometer.

After bonding, the specimens were stored in distilled water at $37^{\circ} \mathrm{C}$ for $24 \mathrm{~h}$. At the end of this period the specimens were debonded by using a universal testing machine (EMIC, line DL, series 385, São José dos Pinhais, PR, Brazil) at a crosshead speed of $0.5 \mathrm{~mm} / \mathrm{min}$. The specimens were debonded in a direction parallel to the buccal surface. An occlusogingival load was applied to the tubes to produce a shear force at the tooth/ tube interface until fracture occurred. A computer electronically connected to the test machine recorded the results of each test. To calculate bond strength, the debonding force values $(\mathrm{N})$ were converted to $\mathrm{MPa}\left(\mathrm{N} / \mathrm{mm}^{2}\right)$ by taking into account the surface area data provided by the manufacturer.

Descriptive statistics including the mean, standard deviation, median and minimum and maximum values were calculated for each group of teeth tested.

ANOVA was used to compare the results of the three groups. Tukey's pairwise comparison test was used to determine which groups were statistically different from each other. The results were regarded as significant if the $p$ value was less than 0.05 . These analyses were performed with Statistica software (Statistica for Windows version 6.0; Statsoft, Tulsa, OK, USA).

\section{RESULTS}

The descriptive statistics for the shear bond strength of each of the groups tested are presented in $\mathrm{MPa}$ in Table 1.

ANOVA indicates that the shear bond strength of group 1 (which received an additional adhesive layer on the occlusal tooth/tube interface) was significantly higher $(p<0.05)$ than that of the other groups (Table 1 ). There was no statistically 
significant difference $(p>0.05)$ between groups 2 and 3 in relation to the duration of light-cure (Table 1).

\section{DISCUSSION}

Advances in adhesives have helped reducing the failure rate of orthodontic procedures. Presently, it seems more important to improve clinical operating procedures than to increase the retentive strength of the adhesives currently being used ${ }^{28}$. Several studies have evaluated the failure rate of bonded molars, but only one study has tested an alternative method to reduce the rate of failure ${ }^{8}$. Johnston and McSherry ${ }^{8}$ (1999) evaluated the effect of sandblasting on the bond strength of molar attachments and concluded that sandblasting foil mesh bases is likely to provide only a minimal improvement in clinical performance when bonding molar teeth. As a result, the purpose of the present study was to evaluate a possible alternative technique that could reduce the rate of failure of bonded molars.

Mandibular third molars were selected because, with the evolution of conservative dental treatment, extraction of first and second molars is not frequent. The third molars were relatively easier to obtain. Millet, et $\mathrm{al}^{16}$ (2001) and Retamoso, et al ${ }^{25}$ (2009) also used human third molars to conduct in vitro studies evaluating molar tubes. Despite the possible morphological variations that can be presented in this group of teeth, the selection criteria were applied with extreme precision to eliminate any possible source of undue variation.

The Transbond XT adhesive was selected to bond the tubes because this resin is commonly used in shear bond strength evaluations $s^{3,4,14-17,19,20,25}$.

Initially, the tubes were bonded in all 3 groups according to the manufacturer's instructions. In group 1, an additional layer of adhesive was applied on the occlusal tooth/tube interface immediately after bonding, followed by an additional $10 \mathrm{~s}$ of light-cure (Figures $1 \mathrm{~A}$ and $1 \mathrm{~B}$ ). We opted for $10 \mathrm{~s}$ of light-cure since the light was applied directly to the adhesive; moreover, as outlined in the manufacturer's instructions, this is the time recommended to bond esthetic brackets, since direct illumination of the adhesive throughout the bracket is possible.

To evaluate the results of this alternative method to bond molars, 2 control groups were used. The first control group was group 2 , in which the tubes were bonded traditionally (Figures $2 \mathrm{~A}$ and $2 \mathrm{~B}$ ). However, because the total time of light-cure for group 1 was $30 \mathrm{~s}$, a second control group was included to keep the same light-cure time. Therefore, for group 3, 40 $\mathrm{s}$ after the conventional direct bond, an additional $10 \mathrm{~s}$ of light-cure was applied to the occlusal tooth surface. This 40-s interval corresponds to the mean time that was spent for the application of the reinforcement in group 1. Group 3 was established to clarify whether the adhesive reinforcement or the longer illumination time caused the difference in the results. Since group 1 showed the highest shear bond strength and there was no difference between groups 2 and 3 (Table 1), the adhesive reinforcement really was the responsible by the highest values obtained in group 1.

The teeth were etched with $37 \%$ phosphoric acid for $30 \mathrm{~s}$ based on preliminary studies ${ }^{6,7}$ that demonstrated that an etch time of $30 \mathrm{~s}$ is required to produce a satisfactory etch pattern on molar teeth.

The bonding procedures were performed by the same operator using a standardized technique. This is an important consideration, based on data from Millett, et al. ${ }^{15}$ (1999), who showed a significant difference in molar tube survival when the procedure was performed by different operators. According to those authors ${ }^{15}$, this difference could be related to the tube location and non-uniformity in the resin thickness between the enamel and bonding bases.

As in other studies $2,3,6,12,14,16,18,24,25$, the specimens were stored in distilled water at $37^{\circ} \mathrm{C}$ until testing 24 h later. Hajrassie and $\mathrm{Khier}^{4}$ (2007) compared the bond strengths of orthodontic brackets bonded to enamel and debonded at various times (10 $\mathrm{min}$, $24 \mathrm{~h}, 1$ week and 4 weeks) and verified that the bond strength values were not time-dependent. In addition, McCourty, et al ${ }^{14}$ (1991) did not observe significant difference in bond strength results 24 $\mathrm{h}$ and 30 days after a direct bond with Transbond adhesive.

To test shear bond strength, many investigators have used a universal testing machine ${ }^{2-4,14,16,24,25}$ with a crosshead speed of $0.5 \mathrm{~mm} / \mathrm{min}^{3,4,14,24}$, which is the same method used in the present study. A recent study ${ }^{10}$ showed that crosshead speed variations between 0.1 and $5 \mathrm{~mm} / \mathrm{min}$ do not influence debonding force measurements or the mode of failure of brackets bonded to enamel with a composite adhesive.

The amount of adhesive remaining on the enamel surface was not evaluated since this study was testing the proposed reinforcement, not the adhesive system. However, in all specimens in which failure occurred, the failure occurred predominantly at the enamel/adhesive interface; this is commonly observed when bond strengths are higher ${ }^{11}$, as observed in this study.

Despite the controversy about in vitro evaluation ${ }^{13}$, the findings of the present study should be considered important because the application of an additional adhesive layer in specimens was previously tested than in humans, with no damage to any in vivo specimens. To 
validate this proposed method in vivo, it should first be tested in the laboratory ${ }^{25}$.

The results clearly show that the application of an additional adhesive layer in the occlusal tooth/ tube interface provides a better bond strength than the conventional direct bond (Table 1). The values obtained in group 1 were higher than those observed in the other two groups. According to the theory of material resistance, when a force is applied to a body (tube) fixed in another element (tooth) using an adhesive material (resin), the tension ( $T$ ) is calculated as the force applied (F) divided by the contact area (A) between the bodies bonded $(T=F / A)$. Considering that the adhesive is a material with the lowest possible rupture tension, we should increase the surface area in order to increase the shear bond strength of the tube/ adhesive/tooth complex. With this purpose in mind, we applied an adhesive reinforcement. The results showed that the additional adhesive layer created an additional contact area between the tooth and the tube so that the applied force was distributed over a more extended surface area, yielding better results for group 1 .

The results of the control groups (groups 2 and 3 , Table 1) are in agreement with those obtained by Knoll, Gwinnett and Wolff ${ }^{11}$ (1986), who observed a shear bond strength of $11 \pm 4.0 \mathrm{MPa}$ for bonded molars. These results are also close to those observed by Bishara et al ${ }^{1}(1998)(11.8 \pm 4.1 \mathrm{MPa})$.

The increase in light-cure time exposure did not influence the shear bond strength (Table 1), since there was no difference between groups 2 and 3. This fact emphasizes the importance of the results: since light-cure time did not influence bond strength, the higher values obtained in group 1 were achieved by the proposed method of bonding molar tubes.

Clinical studies have demonstrated different failure rates for bonded molar tubes ${ }^{15,19,28}$. These failures can be related to several factors, including possible moisture contamination, heavy occlusal contacts, tube base adaptation to the curved buccal molar surface, non-uniform resin thickness and the age of the patient at the initiation of treatment ${ }^{13,15}$. Proffit, Fields and Nixon ${ }^{22}$ (1983) demonstrated that, in normal jaws, the maximum biting force is around $30 \mathrm{~kg}$. In the present study, the values achieved by group 1 , despite not having attempted this measurement, are closer than those of the control groups (group 2 and group 3; Table 1). Since many of the factors described above cannot be changed by orthodontists, the alternative method to bond molars presented here seems to be the best option available to increase the quality of the direct bond.

In the present study, there were no difficulties in maintaining a dry field for moisture control ${ }^{13}$ or in placing the tubes in the desired position in posterior regions during direct bonding. However, it is important to emphasize that, although none of the three groups was affected by the above mentioned problems, group 1 showed the best results.

Many factors must be accounted for when deciding to bond or to band molars, such as the possibility of an enamel etch, the quality of the adhesive material available to bond, the retentive potential of the attachment surface, the substrate (amalgam, resin, porcelain, enamel, or other metallic alloys) and the clinical necessities (type of movement, height of the clinical crown and use of headgear, lip bumpers, or palatal bars $)^{2,28}$. After critical analysis of these factors, if direct bonding is chosen, the alternative method proposed in this study seems to be effective.

Bonding may also be useful in mandibular second molars since these teeth are also difficult to band, particularly when they are only partly erupted ${ }^{28}$.

Most adhesives are rough enough to easily attract plaque ${ }^{28}$, but the regions where the excess adhesive was placed are easy to clean, and the adhesive was applied far from the gingival margin. Therefore, gingival health will probably not be any worse than when bands are used, and patients can also be told to reinforce oral hygiene in the region to which the adhesive is applied. Boyd and Baumrind ${ }^{2}$ (1992) demonstrated that banded molars in both adolescents and adults had significantly more plaque accumulation and gingival inflammation than bonded molars.

Another important clinical point when using this technique in the lower arch is the necessity to check the contact points in the areas where the reinforcement was applied over the molar tubes. This can be done during or after the bonding procedure.

An enamel fracture during debonding was observed in only one specimen. The fracture occurred in the tooth that had the highest debonding value (24.54 MPa, Table 1). A study that evaluated in vitro and in vivo bond strengths showed that the in vivo group had significantly lower mean bond strength values compared to the in vitro group.

Care should be taken with the interpretation of the results, as the experimental conditions in vitro do not reproduce the oral environment. A long-term clinical evaluation of a larger specimen population is necessary to provide clinically valid data.

\section{CONCLUSION}

The present in vitro findings indicate that the application of an additional adhesive layer on the occlusal tooth/tube interface increased the shear bond strength of the bonded molar tubes. 


\section{ACKNOWLEDGEMENTS}

We gratefully acknowledge Morelli Ortodontia, and 3 M Unitek Orthodontic Products, for providing commercial products. We also acknowledge Inser Indústria, Comércio e Serviços Ltda, Sorocaba, SP, Brazil, for allowing us to use the quality control laboratory to complete this study.

\section{REFERENCES}

1- Bishara SE, Gordan VV, VonWald L, Olson ME. Effect of an acidic primer on shear bond strength of orthodontic brackets. Am J Orthod Dentofacial Orthop. 1998;114:234-7.

2- Boyd RL, Baumrind S. Periodontal considerations in the use of bonds or bands on molars in adolescents and adults. Angle Orthod. 1992;62:117-26.

3- Bradburn G, Pender N. An in vitro study of the bond strength of two light-cured composites used in the direct bonding of orthodontic brackets to molars. Am J Orthod Dentofacial Orthop. 1992;102:418-26.

4- Hajrassie MK, Khier SE. In-vivo and in-vitro comparison of bond strengths of orthodontic brackets bonded to enamel and debonded at various times. Am J Orthod Dentofacial Orthop. 2007;131:384-90.

5- Janson G, Graciano JT, Henriques JF, Freitas MR, Pinzan A, Pinzan-Vercelino CR. Occlusal and cephalometric Class II division 1 malocclusion severity in patients treated with and without extraction of 2 maxillary premolars. Am J Orthod Dentofacial Orthop. 2006;129:759-67.

6- Johnston CD, Burden DJ, Hussey DL, Mitchell CA. Bonding to molars - the effect of etch time (an in vitro study). Eur J Orthod. 1998;20:195-9.

7- Johnston CD, Hussey DL, Burden DJ. The effect of etch duration on the microstructure of molar enamel: an in vitro study. Am J Orthod Dentofacial Orthop. 1996;109:531-4.

8- Johnston CD, McSherry PF. The effects of sanblasting on the bond strength of molar attachments - an in vitro study. Eur J Orthod. 1999;21:311-7.

9- Keim RG, Gottlieb EL, Nelson AH, Vogels DS. 2002 JCO study of Orthodontic diagnosis and treatment procedures. Part 1. Results and trends. J Clin Orthod. 2002;36:553-68.

10- Klocke A, Kahl-Nieke B. Influence of cross-head speed in orthodontic bond strength testing. Dent Mater. 2005;21:139-44. 11- Knoll M, Gwinnett AJ, Wolff MS. Shear strength of brackets bonded to anterior and posterior teeth. Am J Orthod. 1986;89:4769.

12- Linklater RA, Gordon PH. An ex vivo study to investigate bond strengths of different tooth types. J Orthod. 2001;28:59-65.
13- Linklater RA, Gordon PH. Bond failure patterns in vivo. Am J Orthod Dentofacial Orthop. 2003;123:534-9.

14- McCourt JW, Cooley RL, Barnwell S. Bond strength of light-cure fluoride-releasing base-liners as orthodontic bracket adhesives. Am J Orthod Dentofacial Orthop. 1991;100:47-52.

15- Millett DT, Hallgren A, Fornell AC, Robertson M. Bonded molar tubes: a retrospective evaluation of clinical performance. Am J Orthod Dentofacial Orthop. 1999;115:667-74.

16- Millett DT, Letters S, Roger S, Cummings A, Love J. Bonded molar tubes - an in vitro evaluation. Angle Orthod. 2001;71:380-5. 17- Öztoprak MO, Isik F, Sayinsu K, Arun T, Aydemir B. Effect of blood and saliva contamination on shear bond strength of brackets bonded with 4 adhesives. Am J Orthod Dentofacial Orthop. 2007;131:238-42.

18- Oztürk B, Malkoç S, Koyutürk AE, Catalbas B, Ozer F. Influence of different tooth types on the bond strength of two orthodontic adhesive systems. Eur J Orthod. 2008;30:407-12.

19- Pandis N, Christensen L, Eliades T. Long-term clinical failure rate of molar tubes bonded with a self-etching primer. Angle Orthod. 2005;75:1000-2.

20- Pasquale A, Weinstein M, Borislow AJ, Braitman LE. In-vivo prospective comparison of bond failure rates of 2 self-etching primer/adhesive systems. Am J Orthod Dentofacial Orthop. 2007; 132:671-4.

21- Petrone J, Fishell J, Berk NW, Kapur R, Scioti J, Weyant RJ. Relationship of malocclusion severity and treatment fee to consumer's expectation of treatment outcome. Am J Orthod Dentofacial Orthop. 2003;124:41-5.

22- Proffit WR, Fields HW, Nixon WL. Occlusal forces in normal and long-face adults. J Dent Res. 1983;62:566-70.

23- Proffit WR, Tulloch JF. Preadolescent Class II problems: treat now or wait? Am J Orthod Dentofacial Orthop. 2002;121:560-2. 24- Rêgo EB, Romano FL. Shear bond strength of metallic brackets photo-activated with light-emitting diode (LED) at different exposure times. J Appl Oral Sci. 2007;15:412-5.

25- Retamoso LB, Collares FM, Ferreira ES, Samuel SM. Shear bond strength of metallic brackets: influence of saliva contamination. J Appl Oral Sci. 2009;17:190-4.

26- Tung AW, Kiyak HA. Psychological influences on the timing of orthodontic treatment. Am J Orthod Dentofacial Orthop. 1998; 113:29-39.

27- Turbill EA, Richmond S, Wright JL. The time-factor in orthodontics: what influences the duration of treatments in National Health Service practices? Community Dent Oral Epidemiol. 2001;29:62-72.

28- Zachrisson BU. A posttreatment evaluation of direct bonding in orthodontics. Am J Orthod. 1977;71:173-89.

29- Zachrisson BU. Cause and prevention of injuries to teeth and supporting structures during orthodontic treatment. Am J Orthod. 1976;69:285-300. 